\title{
A Mathematical Model of the Heat Transfer and Fluid Flow in AOD Nozzles and its Use to Study the Conditions at the Gas/Steel Interface
}

\author{
A. TILLIANDER, T. L. I. JONSSON"1) and P. G. JÖNSSON \\ Division of Metallurgy, Royal Institute of Technology, SE-100 44 Stockholm, Sweden. \\ 1) Also at MEFOS, SE-971 25 Luleå, Sweden.
}

(Received on March 5, 2001; accepted in final form on May 28, 2001)

\begin{abstract}
Knowledge of process gas parameters in the area where the gas leaves the nozzle and then enters the molten steel in AOD converters is necessary in order to determine the boundary conditions needed to model the converter process. Using a newly developed mathematical model for an AOD nozzle verified by comparison against laser Doppler anemometer measurements, the objective of this study was to predict characteristics of non-isothermal heat transfer and fluid flow at the nozzle for pure oxygen gas injected into an AOD converter. The inlet boundary conditions for the nozzle simulation were taken from plant data. The investigation showed that the thermodynamic and physical phenomena in the region where the gas enters the steel melt cannot be determined if the transformation of kinetic energy of gas into heat is not considered because this would amount to oversight of the influences of bubble frequency, temperature, etc. on the process. The possible ranges of bubble frequency and temperature for the nozzle conditions in the study were also determined.
\end{abstract}

KEY WORDS: AOD; nozzle; modeling; gas characteristics; steel.

\section{Introduction}

The aim of the work documented in this report was to predict the gas characteristics at the nozzle outlet to be used as boundary conditions in a future model of the AOD converter process. Gas injection at high flow rates is used in a wide range of processes within the metallurgical field where gas and solid injection is carried out during a large part of the process. The injected gas could be a reacting gas, such as oxygen or hydrogen, or a stirring gas, such as argon or nitrogen. To simulate these processes mathematically, it is important to acquire information on the conditions at the injection point. The transport phenomena for these nozzle systems have been studied very little. However, such studies are ultimately necessary to understand the local processes taking place where the gas phase enters the steel phase.

The investigation focused on nozzles used in manufacturing stainless steel by the argon oxygen decarburization (AOD) process. An AOD converter is schematically described in Fig. 1. In Scandinavia, a typical AOD converter has a 75-tonne steel capacity. Gas is continuously injected through four or more nozzles located on the sides of the converter. In each of these nozzles the gas flow is often as high as $2300 \mathrm{~kg} /\left(\mathrm{m}^{2} \cdot \mathrm{s}\right)$. One of the main aims of the AOD process is to remove carbon dissolved in stainless steel, since carbon can react with chromium and form chromium carbides that have a negative effect on the steel's material properties. The carbon is oxidized by the injection of oxy- gen gas. To stir the metal and promote homogenization of the steel, inert gases such as argon and nitrogen are also injected through nozzles. By injecting an inert gas it is possible to lower the partial pressure of carbon oxide, which according to thermodynamics enhances the removal of carbon during the oxidation process.

The AOD process has not been investigated to the same extent as other metallurgical processes. However, some studies have been carried out using water modeling ${ }^{1-4)}$ and mathematical modeling. ${ }^{5-8)}$ Masterson $^{1)}$ used a 5-tonne AOD water model to investigate the fluid dynamics and mixing time both for bottom and side blowing. Zhu, Sawada and Igushi $^{5)}$ presented a computational fluid dynamics (CFD) plume model of the AOD process to predict the volume fraction of gas in the melt with nozzle exit con-

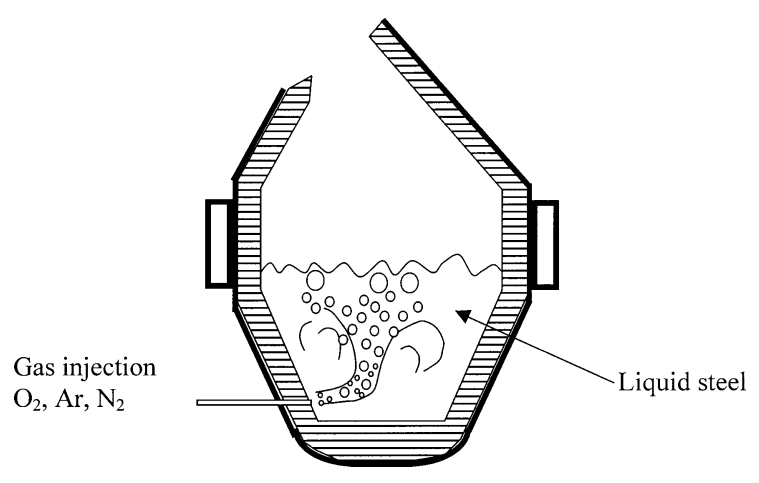

Fig. 1. Sketch of the nozzle and AOD converter. 
ditions approximated using dimensionless numbers, thus not fully covering local processes taking place where the gas phase enters the steel phase. Reports on studies of other types of nozzles can be found in the literature by, for example, Drikakis and Tsangaris ${ }^{9)}$ and Konstantinov and Obermeier. ${ }^{10)}$ These are not, however, relevant in investigating the metallurgical nozzles employed for high gas flows focused on in this study.

In order to verify the model of an AOD nozzle, laser Doppler anemometer (LDA) measurements were performed in co-operation with $\mathrm{AGA} \mathrm{AB}$ using oxygen gas and a stainless steel nozzle with the same dimensions as the inner pipe of the AOD nozzle. The oxygen gas was blown through the nozzle with an inlet pressure of $1 \mathrm{MPa}$ at a flow rate of $400 \mathrm{~nm}^{3} / \mathrm{h}$. This turned out to be the limit of the gas supply system. Heat was applied around the outer boundary in the outlet region of the nozzle to emulate the temperature distribution in a real nozzle during operating conditions. During the experiment the heat source maintained a pipe temperature of $523 \mathrm{~K}$ close to the outlet. The LDA flow detection was done using titanium oxide particles 2 micrometers in diameter. The velocities of the particles were measured just as they left the outlet. The highest velocity, found at the center line of the nozzle, measured $283 \mathrm{~m} / \mathrm{s}$ with a fluctuation of $\pm 25 \mathrm{~m} / \mathrm{s}$. A calculation of these experimental conditions was performed using the nozzle model. The highest calculated gas velocity in the nozzle was $293 \mathrm{~m} / \mathrm{s}$. Thus, the deviation between the measured and calculated velocity was $10 \mathrm{~m} / \mathrm{s}$, i.e. $3.5 \%$, which for these conditions was deemed to be fully satisfactory. A complete description of the experimental study and model verification can be found in a separate report. ${ }^{11)}$ This report covers utilization of the AOD nozzle model for predicting conditions at the gas/steel interface.

In the first part of the paper the physics of gas injection into molten steel are discussed. Next, a mathematical representation of the transport phenomena in nozzles is given. Thereafter, results from the numerical solution of the differential equations are presented and discussed. Finally, the conditions at the gas/steel interface are discussed.

\section{The Physics of Gas Injection into Molten Steel}

\subsection{Physics of the Inner Nozzle Region}

Figure 2 shows the predicted derivative of the velocity curve with respect to distance along the length of the nozzle. The slope of the curve is very steep just before the outlet of the nozzle, which means that it is difficult to calculate the different gas parameters in that region. A high temperature gradient exists along the nozzle length and there is a high expansion of the gas leaving the nozzle. At the nozzle outlet, the steel melt has a temperature of up to $2000 \mathrm{~K}$, but the temperature at the inlet is only about $350 \mathrm{~K}$. Therefore, the usual approach of using an isothermal or adiabatic solution is not appropriate for this system. The gas itself can be assumed to neither absorb nor scatter radiation, since the molecules are relatively small. However, the temperature of the nozzle walls is increased by heat radiated from the steel and this in turn raises the gas temperature, mainly by conduction.

Under these conditions it can be expected that the proper

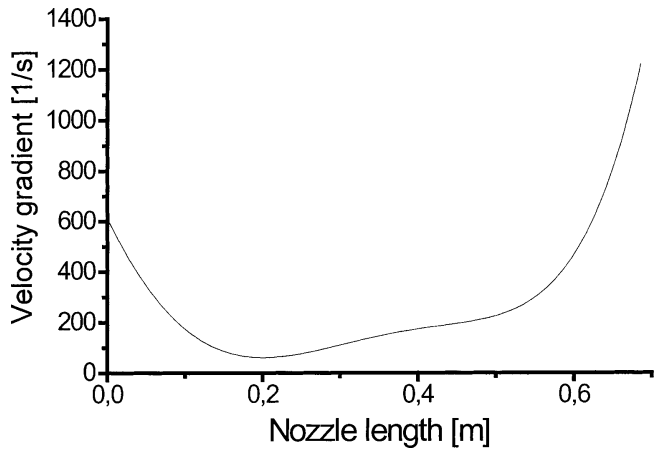

Fig. 2. Velocity derivative as a function of axial distance from the nozzle inlet.

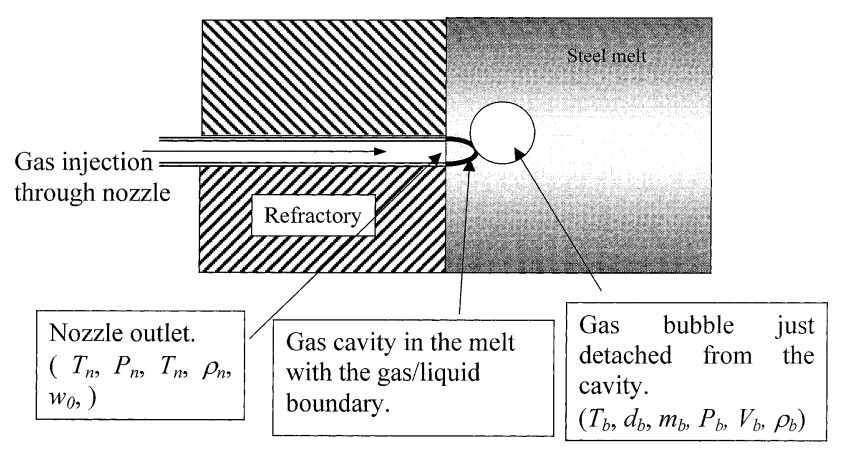

Fig. 3. Sketch of a gas bubble forming in the steel melt at the nozzle outlet.

physical description of the gas is critical for a correct solution of the problem due to the strong coupling between the governing equations for fluid flow and the physics relating the thermodynamic pressure, temperature and density. Furthermore, it could be necessary to include the virial equation of state in order to consider the effect of the volume that the gas molecules occupy on the forces of interaction between the gas molecules. (The oxygen molecule is non-polar but a distortion of the electron distribution could result in temporary polar characteristics ${ }^{12)}$ ).

\subsection{Physics of the Nozzle Exit Region}

\subsubsection{The Physical Picture}

In the first moments of gas injection, the gas stream creates a cavity in the liquid steel just outside the nozzle outlet, ${ }^{13)}$ as schematically shown in Fig. 3. In steady state, the gas leaves the nozzle at a high velocity, close to the local velocity of sound. When the gas molecules approach the melt, the velocity decreases to near zero within a very short distance. ${ }^{14)}$ In this process gas bubbles are formed in the steel melt. ${ }^{15)}$ Thus, the gas $(\sim 0.3 \mathrm{~kg} / \mathrm{s})$ carries a considerable amount of kinetic energy when it leaves the nozzle, which is lost in a very short time close to the gas/steel interface. Chemical reactions certainly also take place, but it takes some time to form bonds and to supply any significant amount of reactants to the region under consideration. Therefore, according to the first law of thermodynamics, the majority of the kinetic energy will be converted to heat. This energy transformation can raise the temperature of the gas just outside the nozzle, raise the temperature of the steel present in the vicinity of the gas/metal interface, or raise the temperature of both the gas and steel in some pro- 
portion simultaneously. To describe this, we introduce $f$, denoting the fraction of the kinetic energy carried by the gas that is converted to heat in the gas phase at the nozzle exit. It should also be noted that when the gas leaves the nozzle it is much colder than the steel and the steel consequently heats the gas.

\subsubsection{The Metallurgical Question}

We would like to be able to fully understand and describe the physics and thermodynamics in the injection region. The chemical reactions along with concentration profiles and the phasic temperature distribution are of greatest interest. At this time, however, the discussion must be confined to rough estimates of what can be expected to happen in the injection area. Therefore, chemical reactions are neglected as a first approximation. However, in the future chemical reactions such as the reaction between carbon and oxygen under the formation of carbon monoxide need to be accounted for.

\subsubsection{Temperature Profiles outside the Nozzle}

Bubble volume data can be obtained from earlier published experiments. ${ }^{16,17)}$ From the calculations performed in this study information is also available regarding the state of the gas when it leaves the nozzle. How this information, together with the introduced factor $f$ (representing the fraction of kinetic energy in gas converted to heat in the gas phase) can be used to calculate the limits for the phasic temperature distribution is presented below.

The mean temperature of the gas bubbles formed at the cavity wall, if only heat transfer from the liquid steel is taken into consideration, $T_{\mathrm{b}}^{\mathrm{ht}}$, can be expressed as follows:

$$
T_{\mathrm{b}}^{\mathrm{ht}}=T_{\mathrm{n}}+\frac{q_{\mathrm{t}}}{m_{\mathrm{b}} C_{\mathrm{p}}^{\mathrm{O}_{2}}}
$$

where $T_{\mathrm{n}}$ is the temperature of the gas at the nozzle outlet and $q_{\mathrm{t}}$ is the total amount of the energy originating from transported heat absorbed by the gas bubble during the time period $t$. The specific heat of oxygen, $C_{\mathrm{p}}^{\mathrm{O}_{2}}$, is expressed as $^{18)}$ :

$$
C_{\mathrm{p}}^{\mathrm{O}_{2}}=1082.03+0.0337 T-\frac{2.4558 \cdot 10^{7}}{T^{2}}
$$

where $T$ is the temperature of the oxygen gas. The mass of the oxygen in one bubble, $m_{\mathrm{b}}$, is related to the density, $\rho_{\mathrm{b}}$, and hence the bubble volume, $V_{\mathrm{b}}$ :

$$
m_{\mathrm{b}}=V_{\mathrm{b}} \rho_{\mathrm{b}}
$$

In order to calculate the bubble volume it is necessary to know the bubble diameter. An average bubble diameter, $d_{\mathrm{b}}$, valid for high flow rates, can be determined using the following empirical equation which has been derived from experiments in air-water and air-oil systems ${ }^{16)}$ and verified for molten steel at $1873 \mathrm{~K}$ by Mori, Sano and Sato: ${ }^{17}$

$$
d_{\mathrm{b}}=0.54\left(V_{\mathrm{g}} d_{\mathrm{n}}^{0.5}\right)^{0.289}[\mathrm{~cm}]
$$

where $V_{\mathrm{g}}$ is the gas flow rate in $\mathrm{cm}^{3} / \mathrm{s}$ and $d_{\mathrm{n}}$ is the nozzle outer diameter in $\mathrm{cm}$. It was found that the bubble size does not depend on the liquid's physical properties at these high flow rates.
With the bubble size given by Eq. (4), the energy transfer from steel to gas due to heat transport as a function of time can be calculated, assuming that only heat conduction takes place. Noting the similarity between diffusion and heat conduction, the equation for diffusion in a sphere ${ }^{18)}$ can be used. For shorter times, the solution is expressed as:

$$
\frac{q_{\mathrm{t}}}{q_{\infty}}=6\left(\frac{\alpha t}{r_{\mathrm{b}}^{2}}\right)^{1 / 2}\left(\pi^{1 / 2}+2 \sum_{n=1}^{\infty} \operatorname{ierfc} \frac{n r_{\mathrm{b}}}{\sqrt{(\alpha t)}}\right)-3 \frac{\alpha t}{r_{\mathrm{b}}^{2}}
$$

where $q_{\infty}$ is the total amount of the energy from heat absorbed by the gas bubble after infinite time, $\alpha$ is the thermal diffusivity and $r_{\mathrm{b}}$ is the radius of the bubble, taken from Eq. (4). The function ierfc is defined as:

$$
\operatorname{ierfc} x=\frac{1}{\pi^{1 / 2}} \exp \left(-x^{2}\right)-x \operatorname{erfc} x
$$

where erfc is the error function complement. The thermal diffusivity is expressed by the function:

$$
\alpha=\frac{k}{C_{\mathrm{p}}^{\mathrm{O}_{2}} \rho_{\mathrm{b}}}
$$

where $k$, the thermal conductivity for oxygen, is a function of temperature: ${ }^{18)}$

$$
k=-2.058 \cdot 10^{-2}+8.254 \cdot 10^{-5} T
$$

So far, only the heat transfer from the steel has been considered. When calculating the initial bubble temperature it is also necessary to take the contribution of the kinetic energy from the oxygen gas per unit time, $E^{\mathrm{kin}}$, into consideration. $E^{\text {kin }}$ can be expressed as:

$$
E^{\mathrm{kin}}=\frac{\dot{m} w_{0}^{2}}{2}
$$

where $\dot{m}$ is the oxygen mass flow and $w_{0}$ is the average axial velocity of the oxygen at the nozzle outlet. The kinetic energy in the gas could be absorbed by the steel surface in the cavity or by the gas while forming a bubble or both cases could occur simultaneously. The energy balance for the steel and gas absorption of the kinetic energy and the surface energy is expressed as:

$$
E^{\mathrm{kin}}=E_{\mathrm{Fe}}+E_{\mathrm{O}_{2}}+E_{\delta} \Rightarrow E_{\mathrm{O}_{2}}=f E^{\mathrm{kin}}-E_{\delta}
$$

where $E_{\mathrm{Fe}}$ and $E_{\mathrm{O}_{2}}$ are the kinetic energy absorbed by the steel boundary layer and the gas phase, respectively. The variable $f$ is the fraction of kinetic energy absorbed by the gas phase and $E_{\delta}$ is the surface energy needed to form the gas-liquid surface. $E_{\delta}$ is calculated from:

$$
E_{\delta}=4 \pi r_{\mathrm{b}}^{2} \delta_{\mathrm{Fe}}
$$

where $\delta_{\mathrm{Fe}}$ is the surface tension of steel.

The fraction of the kinetic energy carried by the gas that is converted to heat in the gas phase at the nozzle exit can be determined using Eqs. (1) to (11). The total energy absorbed by the gas bubble, $E_{\mathrm{O}_{2}}^{\text {tot }}$, can be expressed as: 


$$
E_{\mathrm{O}_{2}}^{\mathrm{tot}}=q_{\mathrm{t}}+f E^{\mathrm{kin}}-E_{\delta}
$$

Since the relationship between $q_{\mathrm{t}}$ and $T_{\mathrm{b}}^{\mathrm{ht}}$ in Eq. (1) must be valid for the total energy absorbed by the gas bubble, the final mean temperature in the gas bubble when it detaches from the cavity could also be expressed as:

$$
T_{\mathrm{b}}^{\mathrm{final}}=\frac{E_{\mathrm{O}_{2}}^{\mathrm{tot}}}{m_{\mathrm{b}} C_{\mathrm{p}}^{\mathrm{O}_{2}}}+T_{\mathrm{n}}
$$

It is thus possible to solve Eq. (13) for any fraction of kinetic energy absorbed by the gas phase, supposing that the state of the gas at the nozzle outlet is known. The very interesting question is: what are the limits of $f$ in Eq. (10)? In order to answer this it is necessary to fully understand the physics and thermodynamics in the injection region. The matter is further discussed in Chapter 4. However, what precisely happens at the gas/steel interface for the conditions under consideration here remains unclear, but will hopefully be revealed in future research.

\section{Mathematical Model}

The oxygen flow in an industrial nozzle from the AOD converter has been studied in this investigation. A schematic picture of a nozzle is given in Fig. 4. The inner nozzle is made of copper and the outer nozzle is made of stainless steel.

\subsection{Mathematical Formulation of the Nozzle}

For the model statement, the following assumptions have

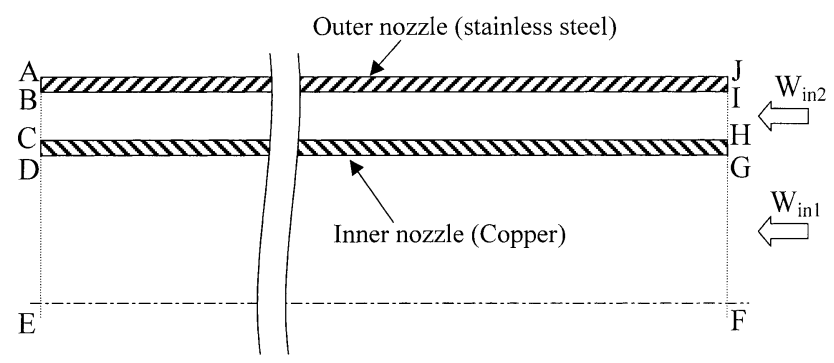

Fig. 4. Outline of the nozzle and region of integration used in the calculations. been made.

- The absorption of radiation is zero for the gas.

- Gravity is neglected.

- The laminar viscosity is a function of temperature only.

- The gas is pure oxygen.

- The gas flow is turbulent. The well-established $k-\varepsilon$ model is used to account for turbulence.

- The gas flow in the nozzle is independent of time (i.e. steady state).

\subsection{Transport Equations for the Nozzle}

The governing transport equations (in two-dimensional cylindrical co-ordinates) to be solved based on the previous assumptions are:

- Conservation of mass

- Conservation of radial and axial momentum

- Conservation of thermal energy

The well-known $k-\varepsilon^{19)}$ model was used to describe the gas turbulence behavior in the nozzle. The effective viscosity is an expression of the laminar viscosity, $\mu_{1}$, and turbulent viscosity, $\mu_{\mathrm{t}}$ :

$$
\mu_{\mathrm{eff}}=\mu_{1}+\mu_{1}
$$

The turbulent viscosity in turn is expressed as:

$$
\mu_{\mathrm{t}}=C_{3} \rho \frac{k e^{2}}{\varepsilon}
$$

where $C_{3}$ is a constant. Its value and the other values of the constants used in the $k-\varepsilon$ model are listed in Table $1 .^{20)}$

\subsection{Boundary Conditions for the Nozzle}

The boundary conditions are summarized in Table 2. The nozzle and the labels used are shown in Fig. 4 (note that figure parts are not proportional).

\subsection{Nozzle Side Walls (AB, CD, GH, and IJ)}

All variables were assumed to have a zero value, except for the enthalpy. $Q_{\mathrm{r} 1}$ is the heat flux due to radiation from the steel bath at $1923 \mathrm{~K}, Q_{\mathrm{r} 2}$ is the heat flux due to the radi-

\begin{tabular}{|c|c|c|c|c|c|c|c|c|c|}
\hline & $\overline{\mathrm{AB}}$ & $\overline{B C}$ & $\mathrm{CD}$ & $\overline{\mathrm{DE}}$ & $\overline{E F}$ & $\overline{F G}$ & $\overline{\mathrm{GH}}$ & $\overline{\mathrm{HI}}$ & $\overline{\mathrm{IJ}}$ \\
\hline $\mathrm{u}$ & 0 & 0 & 0 & 0 & 0 & 0 & 0 & 0 & 0 \\
\hline $\mathrm{w}$ & 0 & $\frac{\partial w}{\partial z}=$ const & 0 & $\frac{\partial w}{\partial z}=$ const & $\frac{\partial w}{\partial r}=0$ & $\mathrm{~W}_{\mathrm{inl}}$ & 0 & $\mathrm{~W}_{\text {in2 }}$ & 0 \\
\hline h & $\frac{\partial h}{\partial z}=Q_{r 1}$ & $\frac{\partial h}{\partial z}=0$ & $\frac{\partial h}{\partial z}=Q_{r 1}$ & $\frac{\partial h}{\partial z}=0$ & $\frac{\partial h}{\partial r}=0$ & $h_{\text {in } 1}$ & $\frac{\partial h}{\partial z}=Q_{r 2}$ & $\mathrm{~h}_{\text {in } 2}$ & $\frac{\partial h}{\partial z}=Q_{r 2}$ \\
\hline ke & 0 & $\frac{\partial k e}{\partial z}=0$ & 0 & $\frac{\partial k e}{\partial z}=0$ & $\frac{\partial k e}{\partial r}=0$ & $\mathrm{ke}_{\text {in } 1}$ & 0 & $\mathrm{ke}_{\mathrm{in} 2}$ & 0 \\
\hline \multirow[t]{2}{*}{$\varepsilon$} & 0 & $\frac{\partial \varepsilon}{\partial z}=0$ & 0 & $\frac{\partial \varepsilon}{\partial z}=0$ & $\frac{\partial \varepsilon}{\partial r}=0$ & $\varepsilon_{\text {in } 1}$ & 0 & $\varepsilon_{\text {in } 2}$ & 0 \\
\hline & & \multicolumn{2}{|l|}{$\mathrm{AJ}$} & \multicolumn{2}{|l|}{$\overline{B I}$} & \multicolumn{2}{|l|}{$\mathrm{CH}$} & \multicolumn{2}{|l|}{$\overline{\mathrm{DG}}$} \\
\hline \multicolumn{2}{|l|}{$\mathrm{U}$} & \multicolumn{2}{|l|}{0} & \multicolumn{2}{|l|}{0} & \multicolumn{2}{|l|}{0} & \multicolumn{2}{|l|}{0} \\
\hline \multicolumn{2}{|l|}{ W } & \multicolumn{2}{|l|}{0} & \multicolumn{2}{|c|}{0} & \multicolumn{2}{|c|}{0} & \multicolumn{2}{|l|}{0} \\
\hline \multirow{2}{*}{\multicolumn{2}{|c|}{$\begin{array}{l}\mathrm{h} \\
\mathrm{ke}\end{array}$}} & \multirow{2}{*}{\multicolumn{2}{|c|}{$\begin{array}{l}\mathrm{h}_{\text {wall }} \\
0\end{array}$}} & \multicolumn{2}{|c|}{$\mathrm{Q}_{\mathrm{rad}}+\mathrm{Q}_{\mathrm{cv}}+\mathrm{Q}_{\mathrm{cd}}=0$} & \multicolumn{2}{|c|}{$\mathrm{Q}_{\mathrm{rad}}+\mathrm{Q}_{\mathrm{cv}}+\mathrm{Q}_{\mathrm{cd}}=0$} & \multicolumn{2}{|c|}{$\mathrm{Q}_{\mathrm{rad}}+\mathrm{Q}_{\mathrm{cv}}+\mathrm{Q}_{\mathrm{cd}}=0$} \\
\hline \multirow{2}{*}{\multicolumn{2}{|c|}{$\mathrm{ke}$}} & & & \multirow{2}{*}{\multicolumn{2}{|c|}{$\begin{array}{l}0 \\
0\end{array}$}} & \multirow{2}{*}{\multicolumn{2}{|c|}{$\begin{array}{l}0 \\
0\end{array}$}} & \multirow{2}{*}{\multicolumn{2}{|c|}{$\begin{array}{l}0 \\
0\end{array}$}} \\
\hline & & \multicolumn{2}{|l|}{0} & & & & & & \\
\hline
\end{tabular}

Table 1. Constants used in the turbulence equations. ${ }^{21)}$

\begin{tabular}{lllll}
\hline$\sigma_{\mathrm{k}}$ & $\sigma_{\varepsilon}$ & $\mathrm{C}_{1}$ & $\mathrm{C}_{2}$ & $\mathrm{C}_{3}$ \\
\hline 1.0 & 1.314 & 1.44 & 1.92 & 0.09 \\
\hline
\end{tabular}

Table 2. Boundary conditions. 
ation from the inlet at $293 \mathrm{~K}$, where $Q_{\mathrm{r} 1,2}=e \Phi\left(T^{4}-T_{\text {surr }}^{4}\right)$. The term e is the emissivity and was assigned the value of 0.5 for both copper (CD and $\mathrm{GH})$ and stainless steel (AB and IJ). (The emissivity is between 0.15 to 0.95 for both materials, depending on the degree of oxidation and grain size). ${ }^{21)}$ The symbol $\Phi$ is the Stefan-Boltzmann's constant, which has a value of $5.6703 \times 10^{-8} \mathrm{~W} / \mathrm{m}^{2} \mathrm{~K}^{4}$.

\subsection{Gas (EF)}

At the axis of symmetry, well accepted boundary conditions were used: zero radial velocity at the axis and zero gradients of all other variables normal to the axis.

\subsection{Nozzle Outlets (BC, DE)}

The dynamic pressure was set at 3.85 bars. Since this boundary could not be obtained, an iteration of the dynamic pressure was carried out. The iteration was terminated when the difference between the preset dynamic pressure at the outlet and the dynamic pressure in the next to the last cell was linear. The gradients for the other variables were zero.

\subsection{Nozzle Inlets (FG and HI)}

The inlet FG is labeled as number 1 and inlet $\mathrm{HI}$ as number 2 . The inlet velocities, $W_{\text {in } 1}$ and $W_{\text {in2 }}$, were calculated from the mass flow of each inlet $\left(2339\right.$ and $95 \mathrm{~kg} / \mathrm{m}^{2} \mathrm{~s}$ for inlet 1 and 2, respectively), which resulted in 139.2 and $11 \mathrm{~m} / \mathrm{s}$, respectively. The radial velocity was assumed to be zero. The inlet temperature of the gas was set to $293 \mathrm{~K}$.

\subsection{Outer Nozzle Wall (AJ)}

All variables had a value of zero except for the enthalpy. It was assumed that the heat from the surrounding lining, $h_{\text {wall }}$, is a linear function based on the corresponding linear variation in the temperature range of $493 \mathrm{~K}$ in the inlet region to $1473 \mathrm{~K}$ in the outlet region.

\subsection{Inner Nozzle Walls (BI, CH, and DG)}

All variables had a value of zero except for the enthalpy. At the walls, the sum of heat fluxes is equal to zero, where $Q_{\text {rad }}$ is the radiation contribution, calculated in respect to the distance from the outlet and inlet. The variable $Q_{\mathrm{cv}}$ is the heat flux due to convection, calculated with the logarithmic law of the wall function, and $Q_{\mathrm{cd}}$ is the heat flux due to conductivity in the material. The thermal conductivity was given the constant values of 381 and $43 \mathrm{~W} / \mathrm{m} \mathrm{K}$ for copper and steel, respectively. The specific heat for the steel was assigned the value of $473 \mathrm{~J} / \mathrm{kg} \mathrm{K}$, and for copper the following expression was used:

$$
C_{\mathrm{p}}^{\mathrm{Cu}}=366.48+9.88 \cdot 10^{-2} T
$$

\subsection{Gas Properties}

Pure oxygen was used in all calculations. The laminar kinematic viscosity data was calculated using the following expression obtained from tabulated values: ${ }^{22}$ )

$$
\mu_{1}=-9.857 \times 10^{-6}+6.232 \times 10^{-8} T+6.945 \times 10^{-11} T^{2}
$$

The density of oxygen was calculated both using the ideal gas law and the equation of state. When the ideal gas law was used, the density was calculated as follows:
Table 3. Constants used in calculating the virial coefficients for oxygen. ${ }^{24)}$

\begin{tabular}{lllll}
\hline $\mathrm{b}_{1}$ & $\mathrm{~b}_{2}$ & $\mathrm{~b}_{3}$ & $\mathrm{~b}_{4}$ & $\mathrm{~b}_{5}$ \\
\hline 0.143389 & -0.629863 & $-5.577814 \times 10^{6}$ & $6.95858 \times 10^{9}$ & $-2.46023 \times 10^{10}$ \\
\hline & & & \\
\hline $\mathrm{c}_{1}$ & $\mathrm{c}_{2}$ & $\mathrm{c}_{3}$ & \\
\hline $4.51336 \times 10^{-3}$ & $9.87169 \times 10^{10}$ & $-3.64928 \times 10^{12}$ \\
\hline
\end{tabular}

$$
\rho=\frac{M}{R} \frac{\left(P_{\mathrm{d}}+P_{\mathrm{ffe}}+P_{\mathrm{atm}}\right)}{T}
$$

where $M$ is the molar weight of the oxygen gas, $R$ is the gas constant, $P_{\mathrm{d}}$ is the calculated dynamic pressure, and $P_{\mathrm{ffe}}$ is the calculated ferrostatic pressure for an industrial 75-tonne AOD converter (here 1.18 bar). $P_{\text {atm }}$ is the atmospheric pressure, which was set to 1.013 bars.

Wagner, Ewers and Schmidt ${ }^{23)}$ proposed an equation of state for oxygen, truncated after the third virial coefficient since higher virial coefficients than the third can be neglected in the range of this equation. Their equation of state is a power series of density, expressed as:

$$
P=R T \rho\left(1+B(T) \rho+C(T) \rho^{2}\right)
$$

where $B(T)$ and $C(T)$ are the second and third virial coefficients. The coefficients were fitted to experimental data for oxygen in the range of: $68 \leq T \leq 406 \mathrm{~K}$ and $0 \leq \rho \leq 6.815$ $\mathrm{mol} / \mathrm{dm}^{3}$ (i.e. $218 \mathrm{~kg} / \mathrm{m}^{3}$ ). Their experimental values can be expressed as:

$$
\begin{aligned}
& B(T)=b_{1}+b_{2} T^{-0.25}+b_{3} T^{-3.5}+b_{4} T^{-4.5}+b_{5} T^{-5.5}\left[\mathrm{dm}^{3} / \mathrm{mol}\right] \\
& C(T)=c_{1} T^{-0.25}+c_{2} T^{-6}+c_{3} T^{-6.75}\left[\mathrm{dm}^{6} / \mathrm{mol}^{2}\right]
\end{aligned}
$$

where $b_{1}$ to $b_{5}$ and $c_{1}$ to $c_{3}$ are constants, specified in Table 3. Instead of having the pressure as a function of density, as in Eq. (19), the density needs to be expressed as a function of pressure. In brief, the density was expressed as the inverse of the molar volume. Eq. (22) was used to express the density as a function of pressure using an approach reported on in the literature. ${ }^{24,25)}$

The final equation of state used in the model to predict the density variation is then expressed as:

$$
\begin{gathered}
\rho= \\
R T+B^{*}(T)\left(P_{\mathrm{d}}+P_{\mathrm{ffe}}+P_{\mathrm{ffe}}+P_{\mathrm{atm}}\right)+\left[\frac{C^{*}(T)}{R T}\right]\left(P_{\mathrm{d}}+P_{\mathrm{ffe}}+P_{\mathrm{atm}}\right)^{2}
\end{gathered}
$$

The corresponding coefficients are:

$$
B^{*}(T)=B(T) \quad \text { and } \quad C^{*}(T)=C(T)-B(T)^{2}
$$

\subsection{Method of Solution}

The solutions of the governing transport and turbulence equations and boundary conditions were obtained using the commercial software PHOENICS. ${ }^{26)}$ PHOENICS is a finite difference code. During a calculation the difference equations were solved by iteration until the difference in the residuals was less than $1 \%$. A typical calculation used a 


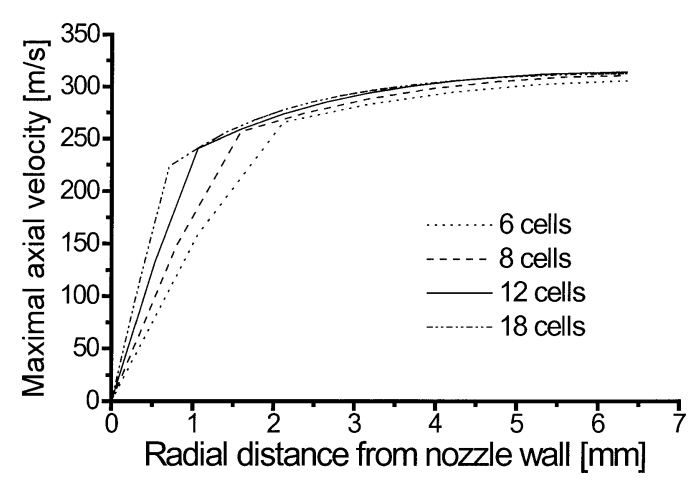

Fig. 5. The maximum velocity at the nozzle exit as a function of radial distance from the nozzle wall. Predicted values are given for the following number of cells in the radial direction: $6,8,12$ and 18 .

$26 \times 300$ grid, of which 12 of the 26 cells in the radial direction represented the inner nozzle radius. The calculation grid was non-uniform and required $8 \mathrm{hr}$ of CPU time on a Sun Sparc station.

\section{Results and Discussion}

\subsection{Gas in the Nozzle}

Calculations were performed for nozzles corresponding to those used in a 75-tonne AOD converter. The length of the nozzle is $686 \mathrm{~mm}$ and the inner diameter is 12.7 and $17.63 \mathrm{~mm}$ for the inner and outer nozzle, respectively. The outer diameter diameter is 14.29 and $19.05 \mathrm{~mm}$ for the inner and outer nozzle, respectively. Pure oxygen was used in all calculations. A typical gas flow from plant data was chosen: $2339 \mathrm{~kg} /(\mathrm{m} \cdot \mathrm{s})$.

Figure 5 shows the effect of the number of cells in the radial direction on the axial velocity in the inner nozzle. The axial velocity at the inner nozzle outlet is plotted as a function of the distance from the nozzle's copper wall. The axial velocity profiles differ significantly in the different cases of radial distances smaller than $2 \mathrm{~mm}$ from the copper nozzle wall. However, for distances larger than $2 \mathrm{~mm}$, the axial velocities only differ by up to $5 \%$ for a variation of 6 to 18 cells in the radial direction. The discontinuous point in the curve at the boundary point between the nearest cell and the next cell to the wall is due to that the logarithmic law of the wall was used. It was ensured that the dimensionless wall distance in the first cell was between 30 and 130, which according to our experience makes the logarithmic law of the wall applicable.

It was also of interest to compare the average axial velocity at the nozzle outlet since that could be used as a boundary condition in the future for the mathematical modeling of different metallurgical reactors. The average axial velocity is plotted against the number of cells in the radial direction in Fig. 6. The value is $4.1 \%$ higher when 18 cells are used compared to when 6 cells are used. However, the difference is only $0.4 \%$ when 18 cells are used instead of 12 . The maximum axial velocity as a function of the number of cells in the radial direction can be seen to increase with an increased number of cells up to 12 cells and then decrease slightly at 18 cells.

Due to the uncertainty of the emissivity value, a compar-

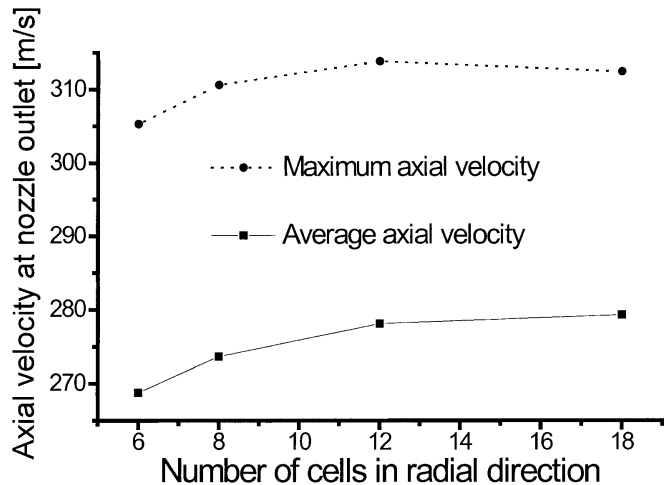

Fig. 6. The velocity versus the number of cells in the radial direction. Data are given for both the average and maximum velocities at the nozzle outlet.

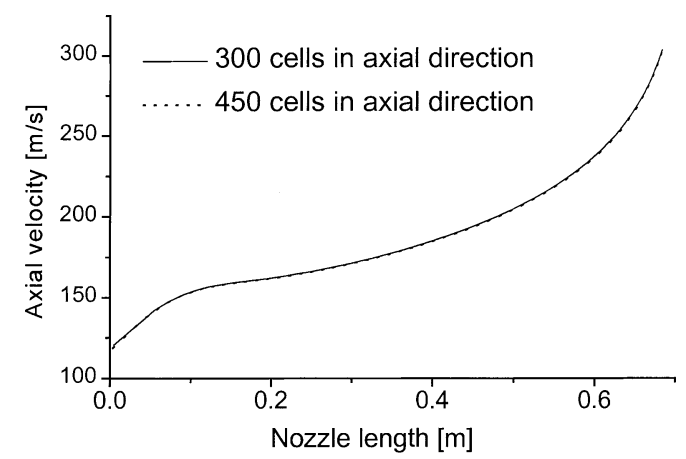

Fig. 7. The axial velocity at the center of the nozzle as a function of axial distance from the nozzle inlet. Data are given for two cases, 300 and 450 cells in the axial direction.

ison with a different value of the emissivity for copper and steel was made. The emissivity was changed from 0.5 for both materials to 0.7 for copper and 0.3 for steel. The difference in the predicted values was expected to be most affected by the copper emissivity. However, the predicted values from the two cases made no noticeable changes in the calculated velocity at the outlet. The only difference was in the temperature, which increased by $0.3 \%$ at the copper wall, but no effect could be determined for the temperature at the center line of the nozzle outlet.

The variation in the axial velocity along the nozzle was also of interest in the study. Figure 7 illustrates the variation in axial velocity with nozzle length at the center of the nozzle. The axial velocity increases steadily up to approximately $600 \mathrm{~mm}$ from the nozzle inlet. Thereafter, it rapidly increases closer to the outlet. In Fig. 7 the effect of using fewer cells in the axial direction is also illustrated. Two different cases were calculated, with 450 and 300 cells in the axial direction. The difference in velocities between the two cases was very small and did not exceed $0.01 \%$ at any location along the center line of the nozzle. Also, two different cell sizes near the nozzle outlet were compared. The first case had the same grid size along the nozzle, and the second case had a decreasing grid size from the nozzle inlet towards the nozzle outlet, resulting in the smallest cell sizes being 1.52 and $1.1 \mathrm{~mm}$, respectively. The predicted axial velocities differed only by a maximum of $0.4 \%$ between the two cases.

In Fig. 8, the density of the oxygen gas at the center of 


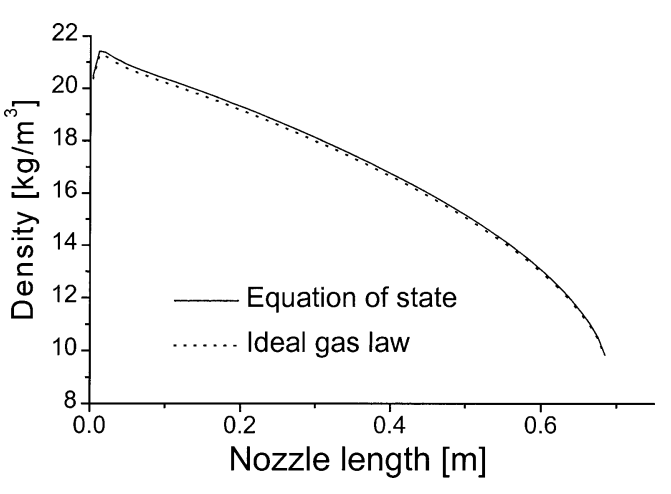

Fig. 8. Density at the center of the nozzle as a function of axial distance from the nozzle inlet. Data are given for two cases: a) using the ideal gas law and b) using the real gas law.

Table 4. Results from the calculation showing the average gas property values at the nozzle outlet.

\begin{tabular}{ccccc}
\hline $\begin{array}{c}\mathrm{W} \\
(\mathrm{m} / \mathrm{s})\end{array}$ & $\begin{array}{c}\rho \\
\left(\mathrm{kg} / \mathrm{m}^{3}\right)\end{array}$ & $\begin{array}{c}\mathrm{T} \\
(\mathrm{K})\end{array}$ & $\begin{array}{c}\mathrm{ke} \\
\left(\mathrm{m}^{2} / \mathrm{s}^{2}\right)\end{array}$ & $\begin{array}{c}\varepsilon \\
\left(\mathrm{m}^{2} / \mathrm{s}^{3}\right)\end{array}$ \\
\hline 261 & 8.86 & 268 & 747 & $1.32 \times 10^{\top}$ \\
\hline
\end{tabular}

the nozzle is plotted against the axial distance from the nozzle entrance. The density decreases with the distance from the nozzle inlet. Resulting differences from using the ideal and the real gas law for the gas density are also shown in the figure. It can be seen that there is little difference between the two cases. More specifically, the maximum difference is only $0.7 \%$. In general, the resultant difference in calculating the oxygen gas density using the two different methods increases with a higher pressure and a lower temperature for the studied conditions. However, the gas temperature is high in the same region where the pressure is high and vice versa. Therefore, in comparing predictions done using both methods, it appears that the effect of temperature on density is counteracted by the effect of pressure.

\subsection{State of the Gas Leaving the Nozzle Outlet}

The results from the calculations are shown in Table 4 as average values from the next to last cell row at the nozzle outlet. When comparing the predicted axial velocity with experimental results by Hoefele and Brimacombe it is clear that the flow is near a steady jetting behavior, as shown in Fig. 9, which makes the assumption of steady state valid and appropriate. ${ }^{27}$ ) The work done by Hoefele and Brimacombe showed that the modified Froude number and the density ratio between fluid and gas could describe the jet behavior. Three regions were defined in their study: a pulsing region at low flow rates where the fluctuation of pressure was regular, a steady jetting region at high flow rates with few irregular pulses or practically steady pressure, and the transition region of irregular fluctuation between the two previous regions. The isotherms of the gas at the outlet are shown in Fig. 10. The predicted results illustrate that the temperature decreases faster as the velocity slope increases at the outlet.

\subsection{Gas/Liquid Boundary}

The calculated values from the simulation of the nozzle itself have been used to get an idea of the physical condi-

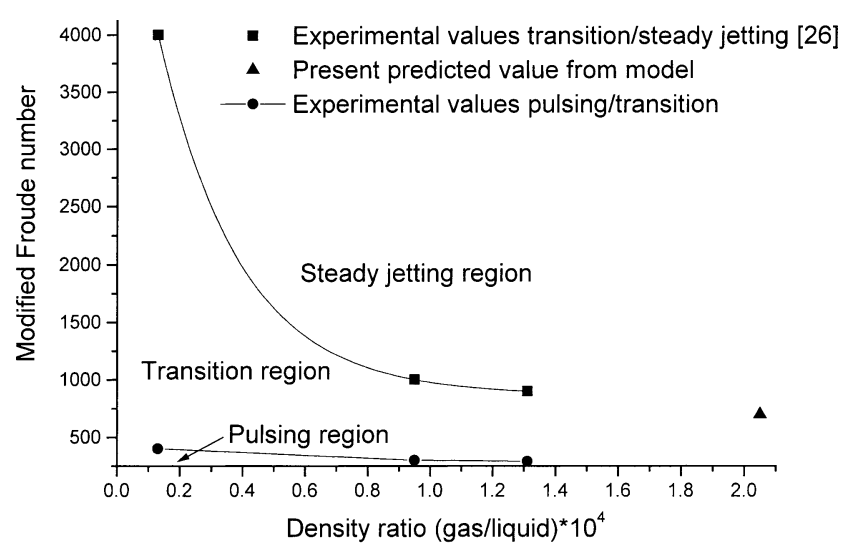

Fig. 9. The model-predicted value of the modified Froude number and the experimental values from Hoefele and Brimacombe for changing type of flow region. ${ }^{27)}$

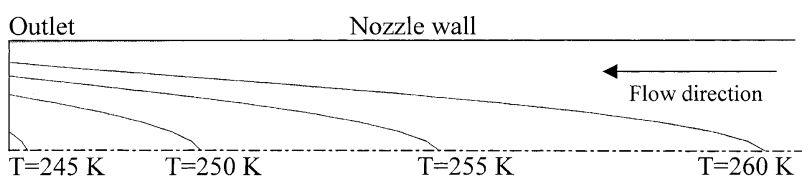

Fig. 10. Isotherms at the nozzle outlet (the last $30 \mathrm{~mm}$ of the nozzle).

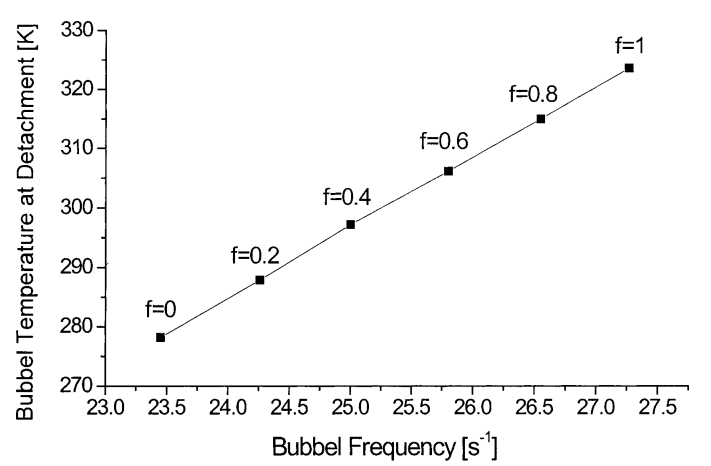

Fig. 11. Gas temperature in a bubble at the nozzle outlet and bubble frequency as a function of the fraction of energy that is transferred to the gas phase.

Table 5. Constants used for calculation.

\begin{tabular}{llll}
\hline$r_{n}$ & $\mathrm{P}_{\mathrm{b}}$ & $\delta_{\mathrm{Fe}}{ }^{18)}$ & $d_{n}$ \\
$(\mathrm{~mm})$ & $(\mathrm{Bar})$ & $\left(\mathrm{J} / \mathrm{m}^{2}\right)$ & $(\mathrm{mm})$ \\
\hline 6.35 & 2.18 & 1.72 & 14.3 \\
\hline
\end{tabular}

tions at the gas/metal interface outside the nozzle. The mean bubble temperature at detachment from the cavity can be calculated using Eq. (13) for different values of the fraction of the kinetic energy, $f$, of the gas that is converted to heat in the gas phase at the gas/metal interface. Also, with a given mass flow, absolute pressure, and the calculated mean bubble temperature and volume, the bubble frequency, bf, can be calculated. In Fig. 11, the results of this calculation are shown as a function of the kinetic energy fraction absorbed by the gas phase. Mean values of the properties during the time of formation of one bubble were used in the calculations. Other values used in these calculations are provided in Table 5. As can be seen, the temperature and bubble frequency increase with an increasing fraction of 
Table 6. Comparison between conductive and convective calculation.

\begin{tabular}{lcccc}
\hline Case & $\begin{array}{c}\mathrm{T}_{\mathrm{b}} \\
{[\mathrm{K}]}\end{array}$ & $\begin{array}{c}\mathrm{bf} \\
{\left[\mathrm{s}^{-1}\right]}\end{array}$ & $\begin{array}{c}\mathrm{T}_{\mathrm{b}} \\
{[\mathrm{K}]}\end{array}$ & $\begin{array}{c}\mathrm{bf} \\
{\left[\mathrm{s}^{-1}\right]}\end{array}$ \\
\hline$f$ & 0 & 0 & 1 & 1 \\
\hline Conduction & 278 & 23.5 & 323 & 27.3 \\
Convection & 350 & 29.5 & 377 & 32 \\
\hline
\end{tabular}

heat contribution from kinetic energy to the gas phase. Further, the range of values could be approximately limited to a certain interval.

It was also interesting to compare the implications of different assumptions regarding the heat transfer mechanism between steel and gas. In Table 6, the results regarding bubble temperature and bubble frequency are given both for the assumption of heat conduction and the assumption of convective heat transfer where data for the extreme cases (where $f$ equals zero and one) were used. In that comparison, $240 \mathrm{~W} / \mathrm{m}^{2} \mathrm{~K}$ was used as the heat-transfer coefficient. Unfortunately, substantial uncertainty regarding the value of this coefficient made the evaluation difficult.

An illustration of some possible implications regarding conditions at the gas/metal interface can be given. If the kinetic energy of the gas were completely absorbed in a thin steel boundary layer, it would be enough to heat such a layer to the temperature of boiling as well as vaporize it. Thus, for the injection of gases into liquids at high flow rates and velocities, this underscores the importance of investigating and carefully considering the conditions at the gas/metal interface outside the nozzle.

\section{Conclusions}

A starting point in the development of process models based on fundamental equations is to determine boundary conditions. For metallurgical reactors using high flow rates and reactive gases, the most critical conditions usually exist in the area where the cold gas meets the liquid metal. For this reason, this study focused on the modeling of conditions in a nozzle used for the injection of a reactive gas into a metallurgical reactor, specifically a nozzle for an AOD converter. The mathematical model of the nozzle was critically tested in different ways:

- A grid sensitivity analysis of the calculation grid showed that the predicted axial velocities were not grid sensitive if the number of cells in the radial direction in the inner nozzle was greater than 12. Furthermore, the number of grid cells in the axial direction did not affect the predicted axial velocities in the outlet region.

- Different gas laws were compared, i.e. usage of the ideal gas law and the virial equation of state in calculating the gas density. The model predictions for the two cases showed only a minor difference. The reason is that the effect of temperature on gas density is counteracted by the effect of pressure. Therefore, it was concluded that it is reasonable to use the assumption of an ideal gas in the model of a nozzle.

- The influence of assuming different emissivity values for the nozzle wall on the calculation results was studied. It was found that the predicted average values at the outlet were only affected by different emissivities to a minor extent.

- The influence of the temperature dependency of phasic properties was investigated. Those properties that were significantly temperature dependent were included in the final model as functions of temperature as described in section III.

- The predicted axial velocities were compared to experimental data of Hoefele and Brimacombe ${ }^{26)}$ and it was shown that the flow is near a steady jetting behavior. This indicates that the steady-state assumption is valid and appropriate.

The calculations focused on the nozzle outlet region since the intention was to use these results as input to process models of metallurgical reactors, such as the AOD converter. However, early in the model's development it was found that the derivative of the velocity curve with respect to distance along the length of the nozzle was very steep close to the nozzle outlet. Thus, the predicted axial velocity was found to increase more rapidly closer to the nozzle outlet and calculation results indicated that the gas leaves the nozzle with a velocity close to the local velocity of sound. This means that a substantial amount of kinetic energy has to be converted to heat within a small volume. Therefore, it was concluded that it is essential to determine the phasic energy distribution within the injection area in order to simulate the metallurgical process accurately.

The present model can be very useful in future research directed towards the development of models simulating, for example, decarburization in an AOD converter.

\section{Acknowledgements}

The authors are grateful for the financial support from the Swedish Steel Producers' Association. We would also like to thank the members of the JK 2335/97 committee for fruitful discussions and AB Sandvik Steel for providing industrial data.

\section{REFERENCES}

1) I. F. Masterson: Process Technology Proceedings, Vol. 6, ISS, Washington D.C., (1986), 377.

2) D. A. Povolotsky, O. K. Tokovoy and S. V. Zyryanov: Russ. Metall. (USA), 1 (1993), 20.

3) R. M. Figueira and J. Szekely: Metall. Trans. B, 16B (1985), 67.

4) P. Blostein, M. Buffenoir, E. Streicher and J. Mallet: Scaninject VI. 6th Int. Conf. on Refining Processes, I, Vol. 2, MEFOS, Luleå, (1992), 129.

5) M. Y. Zhu, I. Sawada and M. Iguchi: ISIJ Int., 38 (1998), 411.

6) O. Wijk and P. Sjöberg: Scaninject VI. 6th Int. Conf. on Refining Processes, II, MEFOS, Luleå, (1992), 5.

7) H. Turkoglu and B. Farouk: ISIJ Int., 30 (1990), 961.

8) P. Gitter, R. Kickinger, S. Pirker, E. Fuhrmann, J. Lehner and J. Steins: Scanmet I, 1th Int. Conf. on Process development in Iron and Steelmaking, Vol. 2, MEFOS, Luleå, (1998), 171.

9) D. Drikakis, and S. Tsangaris: Trans. ASME, J. Fluids Eng., 115 (1993), 115.

10) M. Konstantinov and F. Obermeier: Zeitschrift-für-AngewandteMathematik-und-Mechanik, 79 (1999), S739.

11) A. Tilliander, P. G. Jönsson, T. L. I. Jonsson and S. Lille: KTH, Stockholm Sweden, unpublished research, (2000).

12) D. Tabor: Gases, liquids and solids and other states of matter, 3rd ed., Cambridge University Press, (1991).

13) M. Nilmani and D. G. C. Robertson: Trans. Inst. Min. Metall. C, 89 (1980), C42.

14) G. N. Oryall and J. K. Brimacombe: Metall. Trans. B, 7B (1976), 
391.

15) M. Igushi, M. Kaji and Z. I. Morita: Metall. Trans. B, 29B (1998), 1209.

16) L. Davidson and E. H. Amick, Jr: AIChE J., nr. 2 (1956), 337.

17) K. Mori, M. Sano and T. Sato: Trans. Iron Steel Inst. Jpn., 19 (1979), 553.

18) Handbook of Chemical and Physics, 60th Ed., CRC Press Inc., Florida, (1979).

19) J. Crank: The Mathematics of Diffusion, Oxford Clarendon Press, Oxford, (1956), 86.

20) B. E. Launder and D. B. Spalding: Mathematical Models of Turbulence, Academic Press, London, (1972).

21) E. A. Branders and G. B. Brook: Smithells Metals Reference Book, 7th Ed., Butterworth-Heineman Ltd., Oxford, (1992), 17.
22) Tables of Thermodynamic and Transport Properties of Air, Argon, Carbon Dioxide, Carbon Monoxide, Hydrogen, Nitrogen, Oxygen, and Steam, Pergamon Press, Oxford, (1960).

23) W. Wagner, J. Ewers and R. Schmidt: Cryogenics, 24 (1984), 37.

24) J. H. Dymond and E. B. Smith: The Virial Coefficients of Gases, Clarendon Press, Oxford, (1969).

25) N. A. Gokcen and R. G. Reddy: Thermodynamics, 2nd Ed., Plenum Publishing Corporation, N.Y., (1996), 52.

26) D. B. Spalding: (eds): Recent Advances in Numerical Methods of Fluids, ed. by C. Taylor, K. Morgan Pineridge Press, Swansea, England, (1980), 139.

27) E. O. Hoefele and J. K. Brimacombe: Metall. Trans. B., 10B (1979), 631. 